\title{
Differential Size Distribution and Estrogen Receptor Cargo of Oviductal Extracellular Vesicles at Various Stages of Estrous Cycle in Mice
}

\author{
Chenchen $\mathrm{Yi}^{1} \cdot \mathrm{Ya} \mathrm{Ni}^{1} \cdot$ Peibei Sun ${ }^{1} \cdot \operatorname{Tian}_{\mathrm{Gao}}{ }^{1} \cdot \mathrm{Kun} \mathrm{Li}^{1} \mathbb{D}$
}

Received: 26 August 2021 / Accepted: 20 January 2022 / Published online: 8 February 2022

(c) The Author(s) 2022

\begin{abstract}
Oviductal extracellular vesicles (OEVs) play an important role in fertilization and embryo development. However, it remains largely unknown whether the size and protein cargo of OEVs change during the estrous cycle in mice. This study analyzed the changes in the size distribution and protein cargo of OEVs at four stages of the estrous cycle in mice. The distribution widths of OEVs according to the estrous cycle stage were as follows: proestrus, $20-690 \mathrm{~nm}$ in diameter, with two peaks at 50 and $250 \mathrm{~nm}$; estrus, $22-420 \mathrm{~nm}$ in diameter, with two peaks at 40 and $200 \mathrm{~nm}$; metestrus, 30-70 nm diameter, with a single peak at $40 \mathrm{~nm}$; and diestrus, $10-26 \mathrm{~nm}$ diameter, with a single peak at $20 \mathrm{~nm}$. The estrogen receptor (ER) level in OEVs at the proestrus stage differed significantly from that at estrus $(P=0.013)$ and diestrus $(P=0.005)$. The levels of CD9 and Hsc70 fluctuated across the four stages, although with no significant differences. Furthermore, OEVs were observed among the cilia and microvilli of epithelial cells at the proestrus, estrus, and diestrus stages, but not at the metestrus stage. The number of observed OEVs was the highest at the proestrus stage, followed by the estrus, and the diestrus stage. Endosomes were also observed at the estrus and diestrus stages. The change of the OEV size and ER cargo is associated with the estrous cycle in mice. Our findings increase the understanding of the physiological characteristics of OEVs, which may have clinical applications.
\end{abstract}

Keywords Extracellular vesicles · Exosomes · Oviduct · Oviductal extracellular vesicles · Estrogen receptor · Vesicles size · Estrus

\section{Introduction}

Oviductal extracellular vesicles (OEVs) that are released from the oviduct play important roles in the development of sperm, oocytes, and embryos [1-3]. OEVs have been increasingly documented to have the capacity to advance sperm function and fertilizing ability [4, 5], improve egg maturation and blastocyst yield, and enhance embryo development and quality, via transferring their cargo [6-9]. OEVs carry various biological cargoes, including proteins, mRNA, miRNA, and lipids, and are incorporated into the recipient cells and regulate their development capacity or improve their function; thus, OEVs have potential clinical application as molecular transmitters or therapeutic vectors $[10,11]$.

Kun Li

likun_email@126.com

1 Institute for Reproductive Health, Hang Medical College, Hangzhou 310013, Zhejiang, China
The size distribution and cargoes of OEVs may affect the function and application of exosomes OEVs, for the size of extracellular vesicles is a crucial determinant of their incorporation into recipient cells [12]. The differences in size distribution may be related to the different animal models or methods used [13]. Protein cargoes of OEVs in different species have been revealed $[14,15]$ and OEVs in mice carry various protein markers, including CD9 and Hsc70[16]. However, across the different stages of the estrous cycle in mice, whether the size and protein cargo of OEVs change remain largely unknown. In mammals, the estrous cycle refers to cyclic physiological changes under the action of sex hormones secreted after sexual maturity. In mice, each estrous cycle is divided into four stages: the proestrus, estrus, metestrus, and diestrus stages [17]. The estrous cycle is regulated by endocrine hormones, including prolactin (PRL), luteinizing hormone (LH), follicle-stimulating hormone (FSH), progesterone (P4), and estradiol (E2) [18]. The function and biological roles of the oviduct are regulated by these hormones during the estrous cycle [19, 
20]. Thus, this study aims to provide the characteristics of OEVs for the potential clinical application, based on the hypothesis that the characteristics of OEVs change in mice across the different stages of the estrous cycle.

In this study, we investigated the changes in OEVs at the proestrus, estrus, metestrus, and diestrus stages in mice. We examined the OEV size distribution; changes in the protein cargo carried by OEVs comprising estrogen receptor (ER), CD9, and Hsc70; and the ultrastructure of the OEV-releasing oviduct. The specific components of the OEV protein cargo were selected; the ER is dynamically expressed in the female reproductive tract during the estrous cycle [21]; CD9 is one of the most abundant proteins on the exosomal membrane $[16,22,23]$; and Hsc70 is one of the highly conserved members of the heat shock protein 70 (Hsp70) family; Hsp70 is a known exosomal protein marker and positive control reported in exosome proteomic studies [24, 25]. This study elucidated the characteristics and dynamics of OEVs at different stages of the estrous cycle and thus may be useful for revealing physiological and pathological changes associated with the estrous cycle. It also provides new insights on how to obtain OEVs or extracellular vesicles with uniform size and stable protein cargoes from other sources, which may have clinical applications $[10,11,13]$.

\section{Materials and Methods}

\section{Reagents and Materials}

Rabbit monoclonal anti-CD9 antibody (EPR2949, ab92726; Abcam, Shanghai, China), rabbit monoclonal anti-Hsc70 antibody (EP1531Y, ab51052; Abcam, Shanghai, China), and rabbit monoclonal anti-ER alpha antibody (E115, ab32063; Abcam, Shanghai, Chin $a$ ) were used in this study. Normal rabbit IgG was obtained from R\&D Systems (Minneapolis, MN, USA). Filtered and sterile phosphate-buffered saline (PBS) were purchased from Sigma-Aldrich Corporation (St. Louis, MO, USA). A pre-stained protein ladder was obtained from Thermo Fisher Scientific (Baltics UAB, Vilnius, Lithuania).

\section{Animals}

Female ICR mice aged $>8$ weeks (20-25 g) were purchased from the Experimental Animal Center of Zhejiang Province (License number: SCXK (Zhe) 2019-0002) and Shanghai Jihui Experiment Animal Feeding Limited Co. (License number: SCXK (Hu) 2017-2012). The animals were housed in plastic cages under specific pathogen-free conditions in a temperature-controlled room $\left(23 \pm 2{ }^{\circ} \mathrm{C}\right)$ with $60 \% \pm 10 \%$ relative humidity and a $12 \mathrm{~h}$ light-dark cycle. The study protocol was reviewed and approved by the Animal Ethics Committee of Zhejiang Academy of Medical Sciences. At least 78 mice, ranging from 12 to 34 for different groups, were used for each experiment; the experiments were repeated four times; and all 334 mice were used to obtain sufficient OEVs for further experiments given the small size of the mouse oviduct.

\section{Evaluation of Different Stages of the Estrous Cycle via Vaginal Cytology}

To identify the estrous cycle stage in each mouse, vaginal cells were collected through vaginal lavage. Sterile PBS $(50 \mu \mathrm{L})$ was injected into the vaginal canal and aspirated using a pipette, and the process was repeated 10 times. The aspirated PBS containing vaginal cells were collected into a clean Eppendorf tube, and $10 \mu \mathrm{L}$ of it was placed on a glass slide and covered with a $22 \mathrm{~mm} \times 22 \mathrm{~mm}$ coverslip. The cells were observed under a microscope (Eclipse 80i; Nikon Inc., Tokyo, Japan) at $200 \times$ magnification. Each mouse was classified into one of the four stages (proestrus, estrus, metestrus, and diestrus) as reported [, 17, 18], based on the types and numbers of vaginal cells observed under the microscope. The cell types were classified immediately after sample collection to identify the stage of the estrous cycle. When only epithelial cells with a round nucleus (white arrow with a black edge) were observed, the stage was judged as the proestrus stage (Fig. 1A); when cornified squamous epithelial cells with dense clusters (white arrow) were observed, the estrus stage (Fig. 1B) was identified; when predominantly small leukocytes (black arrow) were seen, the metestrus stage (Fig. 1C) was identified; and when predominantly continuous leukocytes (black arrow), and rarely squamous epithelial cells with fragments and squamous epithelial cells with a round nucleus (white arrow with a black edge), were observed, the stage was judged as the diestrus stage (Fig. 1D). Each mouse was classified into a specific estrous cycle stage for further experiments.

\section{OEV Extraction and Preparation}

After classification into the different stages of the estrous cycle, the mice were euthanized by $\mathrm{CO}_{2}$ overdose. After mincing the oviducts, luminal fluids were collected in sterile and filtered PBS (Sigma) with or without protease inhibitors, as described by Bathala et al. [5]. Clarified luminal fluids were centrifuged at $3500 \times g$ for $10 \mathrm{~min}$ to pellet blood cells and excess tissue debris, and the supernatant was further centrifuged at $14,000 \times g$ for $30 \mathrm{~min}$ at $4{ }^{\circ} \mathrm{C}$ to remove the remaining debris. The supernatant in 10.4-mL polycarbonate bottle with cap (catalog\# 355603, Beckman) was balanced using sterile and filtered PBS (Sigma) and ultracentrifuged at $120,000 \times g$ for $2 \mathrm{~h}$ at $4{ }^{\circ} \mathrm{C}$ using the Beckman Coulter Optima EX-90 ultracentrifuge and the Type $70 \mathrm{Ti} / 70.1 \mathrm{Ti}$ (k-factor 44) rotor. The supernatant was discarded, and the 
Fig. 1 Cell morphological evaluation of vaginal secretions at different stages of the estrous cycle. (A) Proestrus stage: mainly epithelial cells with a round nucleus (white arrow with a black edge). (B) Estrus stage: cornified squamous epithelial cells with dense clusters (white arrow). (C) Metestrus stage: predominantly small leukocytes (black arrow). (D) Diestrus stage: rarely squamous epithelial cells with fragments (black arrow) and epithelial cells with a round nucleus (white arrow with a black edge) but predominantly small leukocytes. The cells were observed under a microscope (Eclipse 80i; Nikon Inc., Tokyo, Japan) with $200 \times$ magnification. Scale bar $=20 \mu \mathrm{m}$
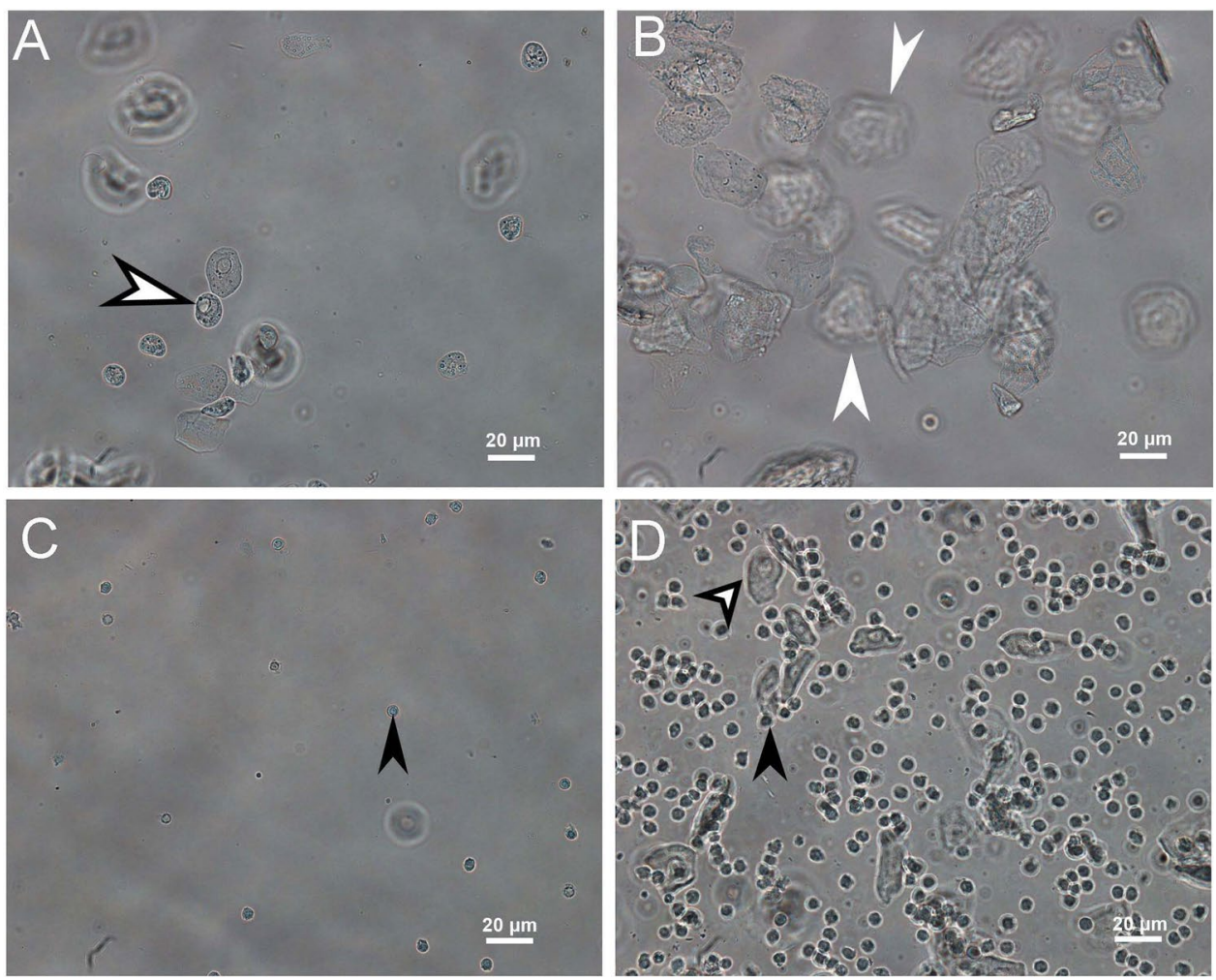

pellets were resuspended in PBS containing a protease inhibitor (Complete Mini EDTA-free Protease Inhibitor Cocktail; Roche Diagnostics Deutschland GmbH, Mannheim, Germany). The protein concentration of the OEV pellet suspension was measured using a bicinchoninic acid (BCA) kit (Beyotime Institute of Biotechnology, Shanghai, China), and the samples were stored at $-80{ }^{\circ} \mathrm{C}$ until use.

\section{Measurement of OEV Size Distribution}

The OEV size distribution was measured using dynamic light scattering on a Zetasizer Nano ZS (ZEN3600) instrument (Malvern Instruments, Malvern, UK), as described by Serrano-Pertierra et al. [26]. The intensity of scattered light at $173^{\circ}$ was measured using a solid-state He-Ne laser at $633 \mathrm{~nm}$. All measurements were performed in triplicate at $25{ }^{\circ} \mathrm{C}$. The samples were diluted with PBS ( $\left.\mathrm{pH} 7.4\right)$ as required. Data processing and analysis were performed using the Zetasizer software, and the results were acquired automatically.

\section{OEV Staining for Transmission Electron Microscopy (TEM)}

OEV staining for TEM was performed according to a method described by Bathala et al. [5]. TEM copper grids (size 200 mesh) with a formvar/carbon film were floated in the OEV suspension, washed with water, and stained with
2\% phosphate tungsten before TEM (HT7700; Hitachi, Tokyo, Japan) imaging and analysis.

\section{Sodium Dodecyl Sulfate-Polyacrylamide Gel Electrophoresis (SDS-PAGE) and Western Blotting}

SDS-PAGE and Western blot analysis were performed as described by Li et al. [27]. The protein concentration of OEVs was measured using a BCA protein assay kit from Beyotime Institute of Biotechnology (Shanghai, China). Equivalent amounts of protein were resolved by SDSPAGE with $10 \%$ acrylamide gels and electrotransferred to Immunoblot-P membranes (Millipore Corporation, Bedford, MA, USA). The membranes were incubated overnight with the anti-CD9, anti-ER, or anti-Hsc70 primary antibodies at $4{ }^{\circ} \mathrm{C}$. Subsequently, the membranes were washed three times (5 min each) using TBS (Tris-buffered saline) containing $0.01 \%(\mathrm{v} / \mathrm{v})$ Tween-20 and incubated with TBS-blocking solution ( $\mathrm{pH} 7.4$ ) containing 5\% non-fat milk for $2 \mathrm{~h}$ at room temperature. Then, the membranes were incubated with a peroxidase-conjugated secondary antibody (1:5000) at room temperature for $1 \mathrm{~h}$, followed by washing three times with TBST. Protein bands on the membranes were probed using a SuperSignal ${ }^{\mathrm{TM}}$ West Femto Maximum Sensitivity Substrate kit (Thermo Scientific, Rockford, IL, USA) following the manufacturer's instructions. The molecular weights of the detected target proteins were inferred using the pre-stained protein markers. The target proteins were quantified via the 
gray intensity of their bands analyzed by ImageJ software and the amount of loaded protein if needed.

\section{Immunoelectron Microscopy}

The immunogold-labeling was performed according to the method described by Bathala et al. [5]. OEVs were mixed with $4 \%$ paraformaldehyde (1:1) for 20 min before being placed on 200-mesh formvar/carbon copper grids. The grids were washed twice with PBS for 3 min each and then washed three times for 3 min with $100 \mu \mathrm{L}$ PBS containing $50 \mathrm{mM}$ glycine. The grids were blocked with $100 \mu \mathrm{L}$ of $5 \%$ bovine serum albumin (BSA) blocking buffer for $30 \mathrm{~min}$ and then incubated with the primary antibodies, anti-CD9 (1:20), and anti-ER (1:20), at $4{ }^{\circ} \mathrm{C}$ overnight. The grids were washed with the buffer for $3 \mathrm{~min}$ (repeated six times) and incubated with the secondary antibody (1:20) labeled with colloidal gold for $30 \mathrm{~min}$. Thereafter, the grids were washed with 5\% BSA-blocking solution (repeated six times), further washed with PBS for $2 \mathrm{~min}$ (repeated six times), fixed with $1 \%$ glutaraldehyde solution for $2 \mathrm{~min}$, and washed with deionized water for 2 min (repeated six times). The OEVs in the grids were negatively stained with uranyl acetate for $90 \mathrm{~s}$ before washing twice with deionized water for $2 \mathrm{~min}$. After drying, the OEVs in the grids were examined with TEM (Tecnai G2 Spirit, Thermo Fisher Scientific) operated at $120 \mathrm{kV}$.

\section{TEM}

TEM was performed as described by Bathala et al. [5]. One oviduct tissue representative of each estrous cycle stage was fixed with filtered PBS containing $2 \%$ formaldehyde and $2 \%$ glutaraldehyde and stored at $4{ }^{\circ} \mathrm{C}$. The tissues were treated with $1 \%$ osmium acid for $1 \mathrm{~h}$, washed three times (10 min each) with water, and treated with $2 \%$ uranium acetate solution. Thereafter, the tissues were dehydrated using the graded series of ethanol $(50 \%, 70 \%, 90 \%, 100 \%$ ethanol) and $100 \%$ acetone. The tissues were then treated with a mixture of embedding agent and acetone (1:1 at room temperature for $2 \mathrm{~h}$, and $1: 3$ at room temperature for $3 \mathrm{~h}$ ). Ultrathin sections of the tissues after polymerization were observed using a Tecnai G2 Spirit electron microscope (Thermo Fisher Scientific) operated at $120 \mathrm{kV}$.

\section{Statistical Analysis}

The relative protein levels are expressed as the mean \pm standard error of the mean. First, the homogeneity of variances of the experimental data was tested; if the $P$-value was $>0.05$, statistical analyses were performed using the least significant difference test, one-way analysis of variance in SPSS 20.0 (IBM, Armonk, NY, USA), or Dunnett's T3 test was performed[27]; if data were non-normally distributed,
Mann-Whitney test was used. Statistical significance was set at $P<0.05$.

\section{Results}

\section{Size Distribution of OEVs Released During the Four Stages of the Estrous Cycle}

The OEV distribution widths were determined to explore the changes in OEV size across the four stages of the estrous cycle. The distribution widths of OEV size at the different stages were as follows: proestrus, 20-690 nm in diameter, with two peaks at 50 and $250 \mathrm{~nm}$; estrus, $22-420 \mathrm{~nm}$ in diameter, with two peaks at 40 and $200 \mathrm{~nm}$; metestrus, $30-70 \mathrm{~nm}$ diameter, with a single peak at $40 \mathrm{~nm}$; and diestrus, 10-26 nm diameter, with a single peak at $20 \mathrm{~nm}$ (Fig. 2, left). TEM was performed to further validate the OEV size. Sphere-like particles were observed, and their size matched the range of distribution widths (Fig. 2, right), suggesting that the OEV size was stable and reliable.

\section{Changes in the Levels of Proteins Carried by OEVs Released at Four Stages of the Estrous Cycle}

To explore the changes in OEV protein cargoes across the four stages of the estrous cycle, the representative OEV cargo proteins, i.e., ER, CD9, and Hsc70, were analyzed using Western blotting and immunoelectron microscopy. The ER is dynamically expressed in the female reproductive tract during the estrous cycle [21] and is a known protein marker. The level of ER in OEVs at the proestrus, estrus, metestrus, and diestrus stages in mice was detected by Western blotting (Fig. 3II). The highest ER level at the diestrus stage was approximately 13-fold higher than the lowest level at the proestrus stage, while the highest ER level at the estrus and metestrus stages was in between these two levels (Fig. 3I). Furthermore, the ER level at the proestrus stage differed significantly from that at the estrus $(P=0.013)$ and diestrus $(P=0.005)$ stages. The presence of ER was confirmed when black round colloidal gold particles were observed on immunoelectron microscopy, and no gold particles were observed when rabbit IgG was used as negative control (Fig. 3III). These results indicated that the ER levels in OEVs changed across the different stages of the estrous cycle.

CD9 levels (Fig. 4II) and Hsc70 levels (Fig. 5II) in OEVs at four stages were detected by Western blotting. The levels of CD9 in OEVs fluctuated across the different stages, and the highest level at the metestrus stage was approximately 2.5 -fold higher than the lowest level at the proestrus stage, whereas the CD9 levels at the estrus and diestrus stages were between these two levels (Fig. 4I); however, the difference in CD9 levels among the stages 

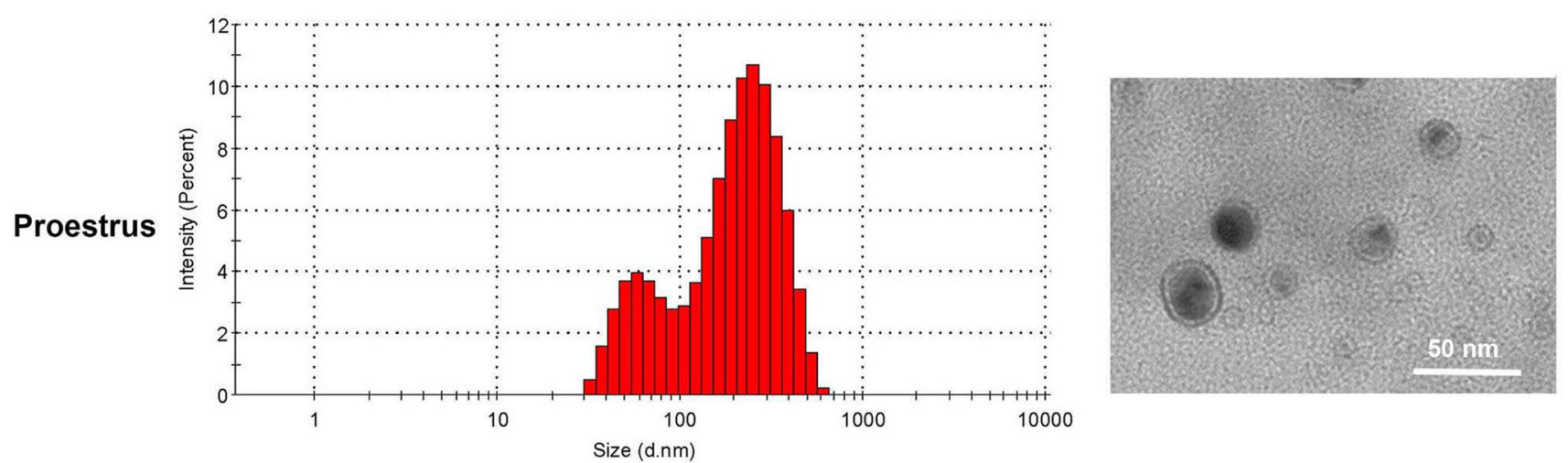

Estrus
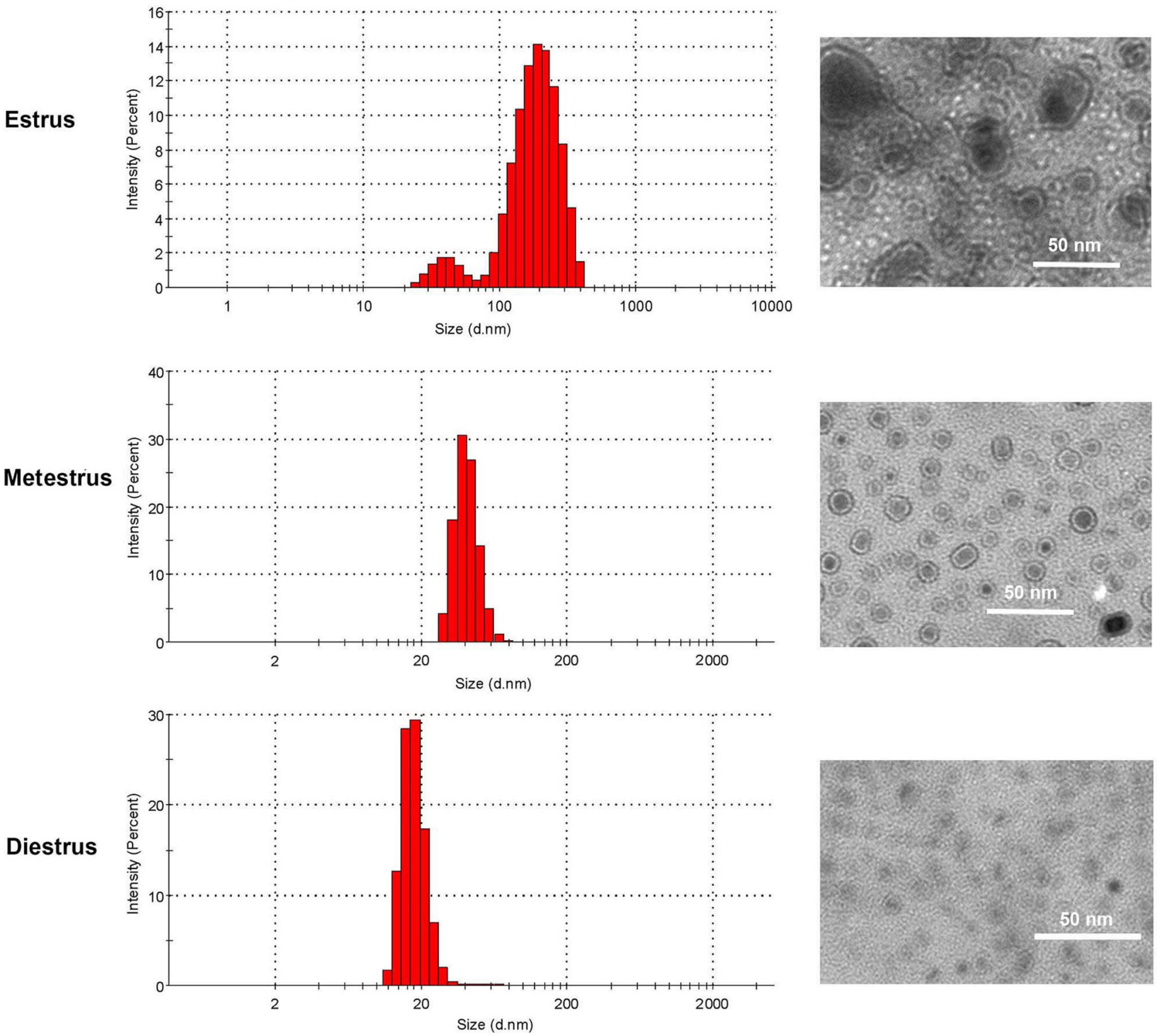

Fig. 2 OEV size characteristics at different stages of the estrous cycle in mice. Left, the size distribution of OEVs; Proestrus OEVs (pooled from 14 mice), Estrus OEVs (pooled from 14 mice), Metestrus OEVs (pooled from 16 mice); Diestrus OEVs (pooled from 34 mice). The graphs illustrating results were automatically acquired using the Zeta sizer software. Right, confirmation of particle size of the corresponding samples on the left via transmission electron microscopic observation. Scale $b a r=50 \mathrm{~nm}$ 
Fig. 3 Quantitative analysis of ER in OEVs at different stages of the mouse estrous cycle. Protein from OEVs, $4.6 \mu \mathrm{g} /$ lane, was loaded when Western blot was analyzed. (I) Quantitative analysis of ER levels in OEVs. The relative levels of ER are determined by standardizing each group with proestrus as 1.00. $* P<0.05$, the ER level at the estrus $(P=0.013)$ and diestrus $(P=0.005)$ stages is statistically different from that at the proestrus stage. Data are presented as mean \pm standard error of the mean, with the experiments repeated three times. (II) Western blot of ER in OEVs. (III) Validation of ER in OEVs using immunoelectron microscopy. (A) Proestrus, (B) estrus, (C) metestrus, (D) diestrus, (E) rabbit IgG control. The scale bar is shown as in the figure; the black gold particles, indicated by the green arrows, indicate that ER in the OEV was recognized by ER antibody

\section{I}

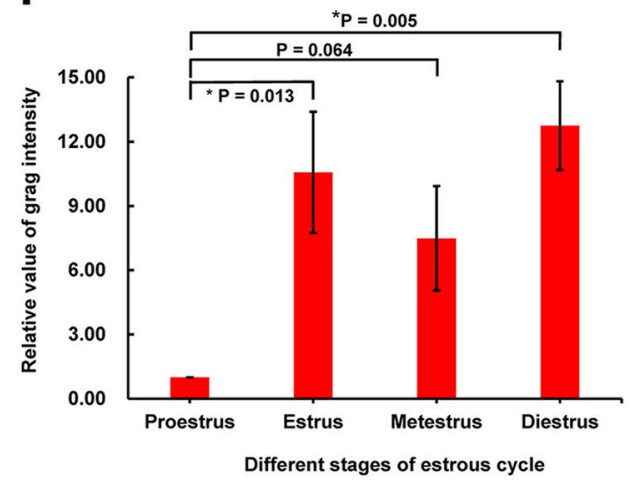

III

A
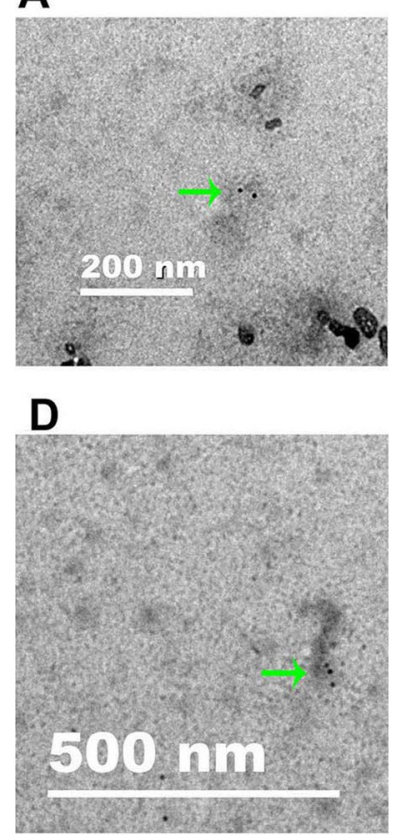

II

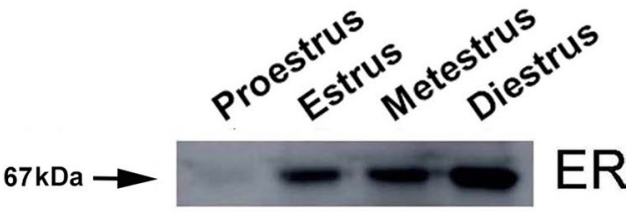

D

B

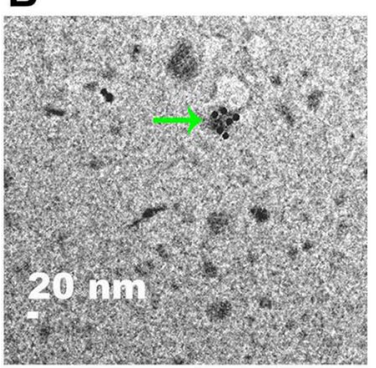

$\mathrm{E}$

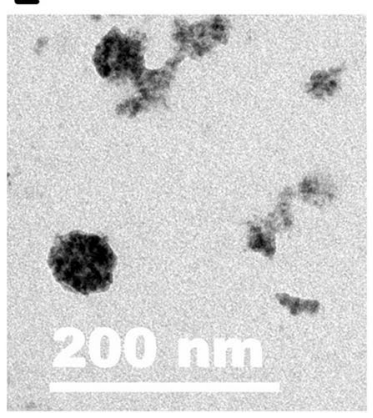

C

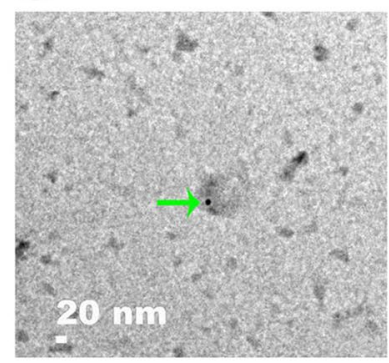

was not significant. The trend of change in the Hsc70 levels was different from that of CD9 but similar to that of the ER. The highest level at the diestrus stage was approximately twofold of the lowest level observed at the proestrus stage, and the level at the estrus and metestrus stages was approximately 1.5 -fold higher than the lowest level at the proestrus stage (Fig. 5I); however, the difference among the stages was not significant. Moreover, black round colloidal gold particles observed on immunoelectron microscopy confirmed the presence of CD9 (Fig. 4III), and no gold particles were observed when rabbit IgG was used as negative control (Fig. 3III). These results indicated that the CD9 level and Hsc70 level fluctuate in OEVs across the different stages of the estrous cycle.

\section{Ultrastructure of the OEV-Releasing Oviduct at Four Stages of the Estrous Cycle}

Oviducts were subjected to TEM analysis to determine the oviduct ultrastructure at different stages of the estrous cycle and examine the status of the released OEVs. At the proestrus stage (Fig. 6A), thick cilia and microvilli were observed on the surface of the epithelial lining of the oviduct lumen, which contained several OEVs (green arrows) of different sizes. Moreover, the number of cilia was the highest, with more cilia than microvilli, at the proestrus stage compared to the other three stages. At the estrus stage (Fig. 6B), similar to the proestrus stage, cilia and microvilli were observed on the surface of the epithelial lining of the oviduct lumen, and OEVs (green arrows) were present among the thick cilia and 


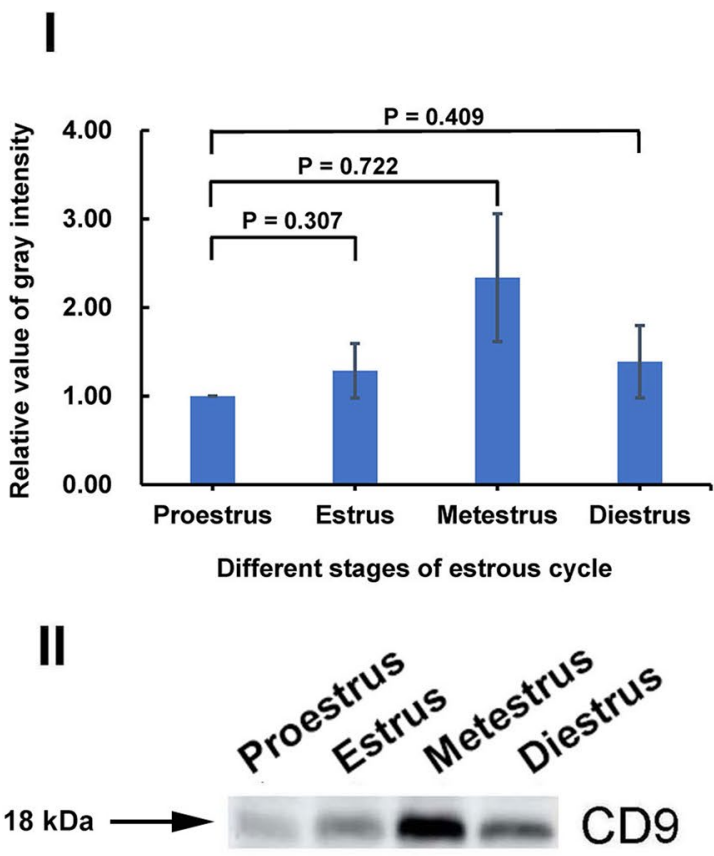

III A

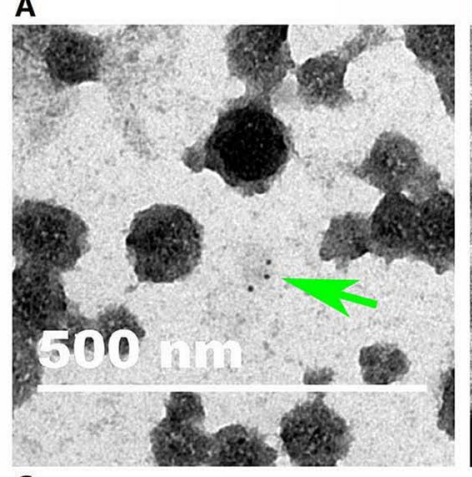

C

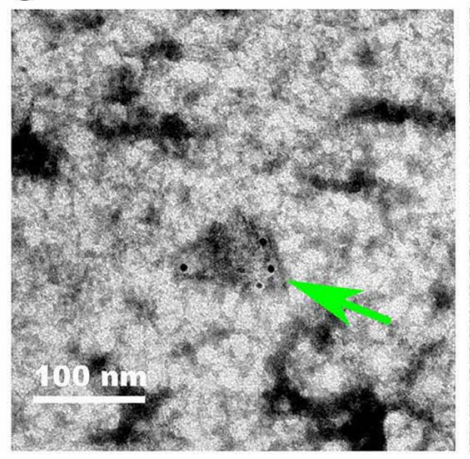

B

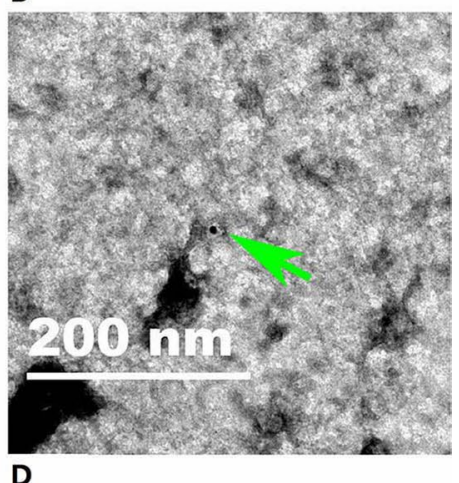

D

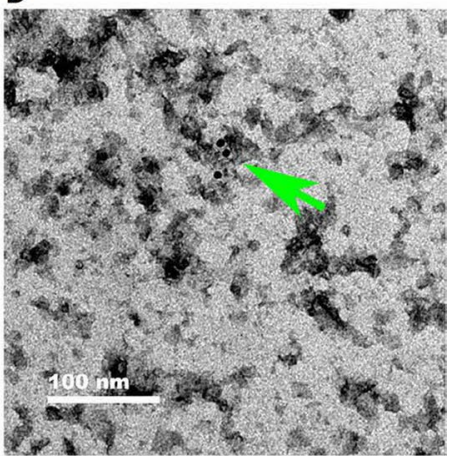

Fig. 4 Quantitative analysis of CD9 in OEV at different stages of the mouse estrous cycle. Protein from OEVs, $4.8 \mu \mathrm{g} /$ lane, was loaded when Western blot was analyzed. (I) Quantitative analysis of CD9 levels in OEV. The relative levels of CD9 are determined by standardizing each group with proestrus as 1.00. Data are presented as mean \pm standard error of the mean, with the experiments repeated six times using three different samples. A $\mathrm{P}$ value less than 0.05 was statistically significant. (II)Western blot of CD9 in OEVs. (III) Validation of CD9 in OEVs using immunoelectron microscopy. (A) Proestrus, (B) estrus, (C) metestrus, (D) diestrus. The observation of dark gold particles indicates the presence of CD9. The scale bar is shown as in the figure; the black gold particles, indicated by the green arrows, suggest CD9 in the OEV were recognized by the CD9 antibody microvilli. Endosomes (yellow arrow) were also observed in blebs. At the metestrus stage (Fig. 6C), only microvilli but no cilia were observed on the surface of the epithelial lining of the oviduct lumen. At the diestrus stage (Fig. 6D), only microvilli but no cilia were observed. OEVs were rarely observed, but endosomes (yellow arrow) were detected. The observed numbers of OEVs at the different stages are shown in Fig. 6E. The difference among the stages was statistically significant, except for metestrus vs. diestrus. These results indicated that the release of OEVs changes across the four stages of the estrous cycle.

\section{Discussion}

To the best of our knowledge, this is the first study to provide evidence that in mice, the size distribution of OEVs changes across the different stages of the estrous cycle. We observed two peaks in the size distribution of OEVs at the proestrus and estrus stages, and only a single peak at the diestrus and metestrus stages. This pattern of OEV size distribution in mice is different from that observed in cattle [24]. In the previous study, the OEV size distribution was $>30 \mathrm{~nm}$, and only one peak was observed in the range of 30-100 nm regardless of the stage of the estrous cycle [24]. In mice in the superovulated cycle, the size distribution of OEVs varies from $<100 \mathrm{~nm}$ in exosomes to $100-1000 \mathrm{~nm}$ in microvesicles [5]. This difference in size distribution in different studies can be attributed to differences in animal models and methods used, as previously reported [12]. Therefore, this study furthers our understanding of how cells produce different sizes of extracellular vesicles, especially when extracellular vesicles are applied [13].

The results of this study suggest that the OEV protein cargoes released under hormone regulation change considerably during the four stages of the estrous cycle in mice. The ER plays a crucial role in endocrine function and reproduction [28], and our results showed that the ER expression level in OEVs changes according to the stage of the natural estrous cycle in mice. The ER is not only present in the nucleus, but also on the plasma membrane [29]; however, the ER may also be detected in the released OEVs because extracellular vesicles are translocated into the cytoplasm via nuclear pores [30]. The physiological significance of the highest level of 

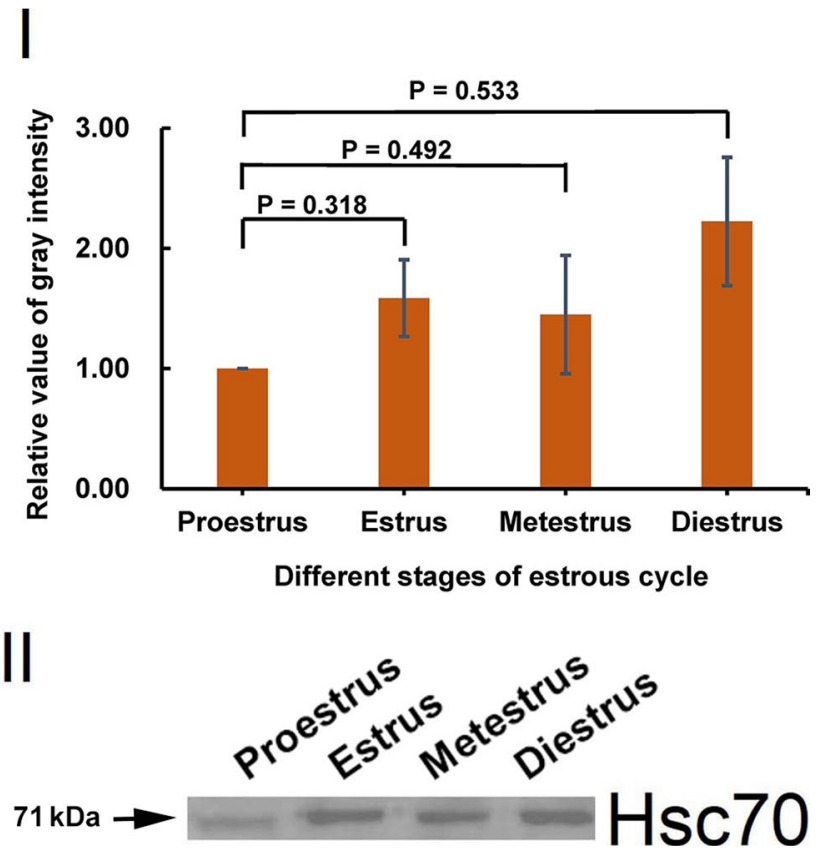

Fig. 5 Quantitative analysis of $\mathrm{Hsc70}$ in OEV at different stages of the mouse estrous cycle. Protein from OEVs, $7.5 \mu \mathrm{g} / \mathrm{lane}$, was loaded when Western blot was analyzed. (I) Quantitative analysis of Hsc70 levels in OEVs. The levels of Hsc70 are determined by standardizing each group with proestrus as 1.00. Data are presented as mean \pm standard error of the mean, with the experiments repeated six times using three different samples. A $P$ value less than 0.05 was statistically significant. (II)Western blot of Hsc70 in OEVs

expression of the ER at the diestrus stage implies that the numbers and the types of the source cells of OEVs, the ciliated and secretory cells [21], may vary, as shown in Fig. 6. Moreover, the CD9 and Hsc70 levels in OEVs fluctuated across four stages, as previously reported [16]. CD9 and Hsc70 are also common exosomes biomarkers [16, 23, 31]. Besides, Hsc70 is also regarded not only as a biomarker but also as a positive control, as shown by Alminana [24]. The results of immunoelectron microscopy showed black gold particles (Figs. 3 and 4), indicating that ER and CD9 in the OEV were specifically recognized by the anti-ER and anti-CD9 antibodies, respectively, although the morphology of OEVs (Figs. 3 and 4) was different from that in Figs. 2 and 6. The OEV morphology is affected by the experiment conditions, including the chemical fixation and contrasting methods [33]. In previous studies using a bovine model, 170 among 336 protein contents of OEVs were differentially abundant across the estrous cycle [24], and several metabolites, including glucose-1-phosphate and methionine that regulate sucrose, glucose, and lactose metabolism, were significantly different across the estrous cycle [24]. Therefore, our results are consistent with the conclusion that the metabolites or contents of extracellular vesicles change during the different stages of the estrous cycle [24, 32].
Currently, variables determining the homogeneity and heterogeneity of the size distribution and composition of vesicles remain unclear, and it is speculated that vesicle size depends on the type of membrane phospholipids and the presence or absence of particular membrane proteins, and the composition may link with the biogenesis pathway and the cells of their origin [34]. However, our results strongly imply that the size distribution and the cargo of OEVs are associated with the physiological status of host cells, as well as influenced by multiple hormones that regulate the estrous cycle because the hormones may control the biogenesis pathway, the resource cells, and further the membrane proteins of OEVs. This result is consistent with a previous study, which showed that estrogen induces increased secretion of smaller-sized vesicles [35]. Additionally, the ciliated and secretory cells in the oviduct at different stages may be influenced by the following hormones in vivo that modulate the morphology of cells in the vaginal canal[18]: the levels of E2 [36] and PRL [37] sharply increase, and LH and FSH release into the circulation $[38,39]$ at the proestrus stage, which is similar to the human menstrual cycle [40]; the levels of FSH and PRL reach the peaks [36, 37] [41, 42], and E2 levels decline $[36,37]$ at the estrus stage; the levels of P4 and E2 start rising [36, 43, 44] at the metestrus stage; and the levels of $\mathrm{P} 4$ reach the peaks $[37,45,46]$ at the diestrus stage. However, the detailed mechanism by which hormones act remains to be further elucidated.

In this study, we successfully observed the status of OEVs released by the oviduct at four stages in the estrous cycle in mice through ultrastructural observation with TEM. The results indicated that the OEV amount is higher at the proestrus and estrus stages and lower at the metestrus and diestrus stages. The observations of the oviduct ultrastructure in this study are consistent with the previously reported features of the murine oviduct [47], including the presence of cilia on the surface of the epithelial lining of ciliated and secretory cells; however, the previous study did not discuss the released extracellular vesicles. It has been reported that the OEVs arise from the apocrine pathway [5]. In this study, we showed the status of OEVs at four stages during the estrous cycle; however, the cilia could not be observed during the metestrus and diestrus stages. Nevertheless, this does not indicate the absence of cilia, because the images showed only a part of one or several cells and not the whole of the oviduct tissue. Based on our results, we propose that the cilia are less common at the metestrus and diestrus stages in mice than those at the proestrus and estrus stages. Thus, the results of this study are consistent with the numbers of the ciliated and secretory cells reported during the estrous cycle in the previous study [48]. Taken together, our study confirmed the release of OEVs from the oviduct and revealed the OEV status during the natural estrous cycle in mice. 
Fig. 6 Ultrastructure of the oviduct and OEV secretion at different stages of the mouse estrous cycle. (A) Proestrus stage: thick cilia and microvilli are seen on the surface of the epithelial lining of the oviduct lumen; many OEVs (green arrows) are present among the thick cilia and microvilli, and the OEV sizes are different. Representative images from a total of 19 photographs. (B) Estrus stage: cilia and microvilli are seen on the surface of the epithelial lining of the oviduct lumen; OEVs (green arrows) are present among the thick cilia and microvilli, and endosomes (yellow arrow) are observed. Representative images from a total of 20 photographs. (C) Metestrus stage: only microvilli but no cilia are observed on the surface of the epithelial lining of the oviduct lumen. Representative images from a total of 14 photographs. (D) Diestrus stage: only microvilli but no cilia are observed. OEVs are hardly observed, but endosomes (yellow arrow) are seen. Representative images from a total of 24 photographs. (E) The number of OEVs in TEM photographs. Data are presented as mean \pm standard error of the mean. $n$, the number of photographs; statistical analyses were performed using Mann-Whitney test. A $P$ value less than 0.05 was statistically significant
A

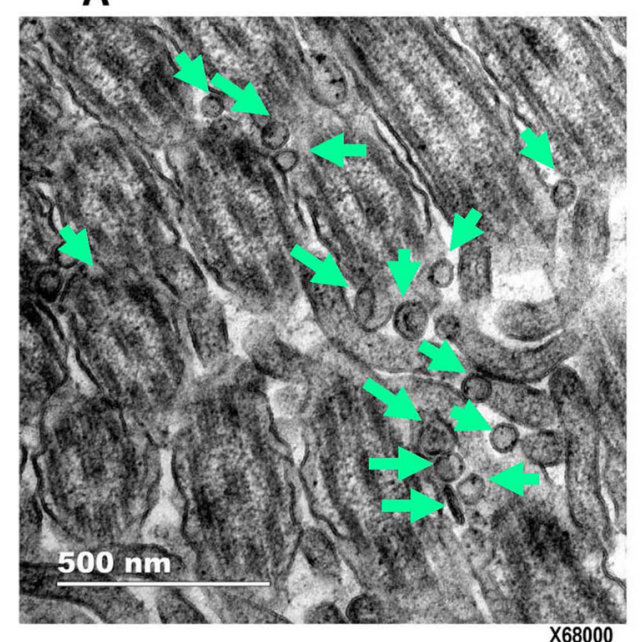

C

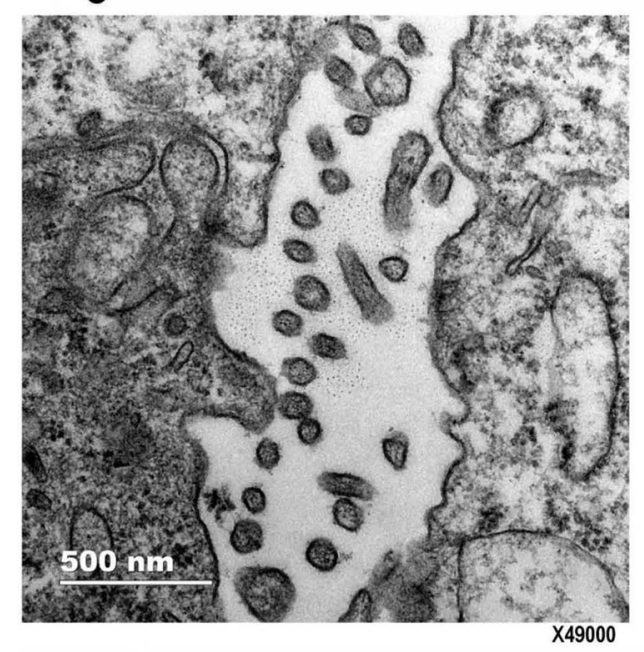

B

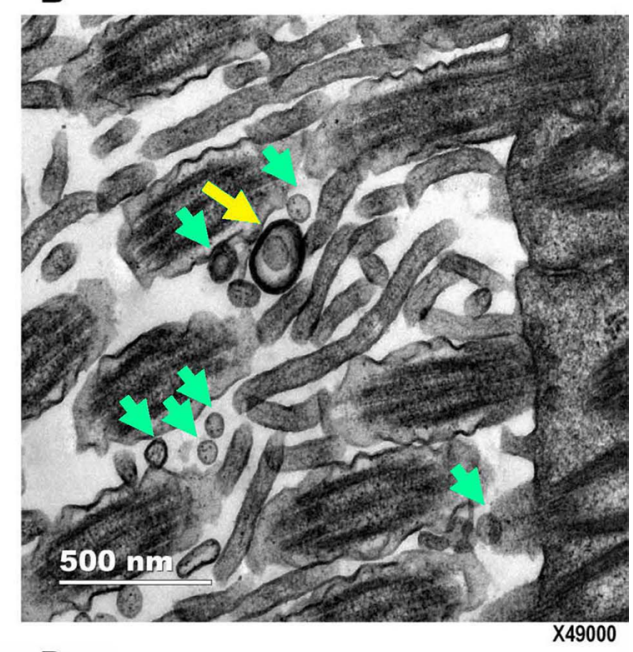

D

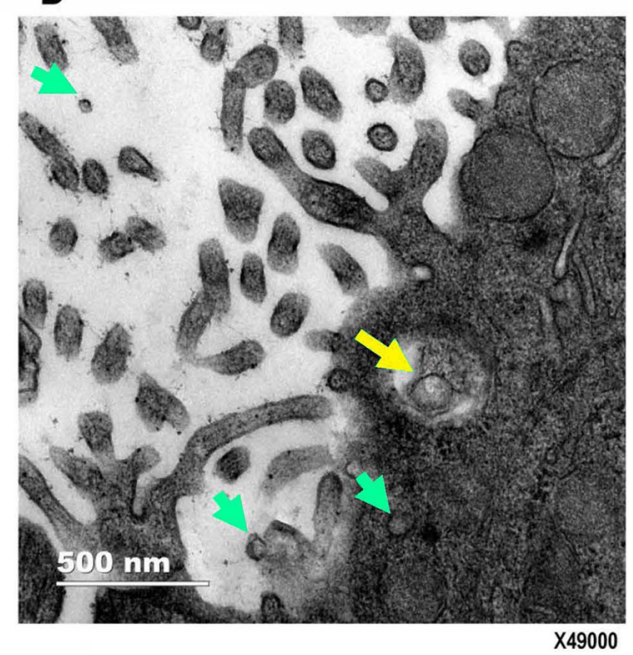

E

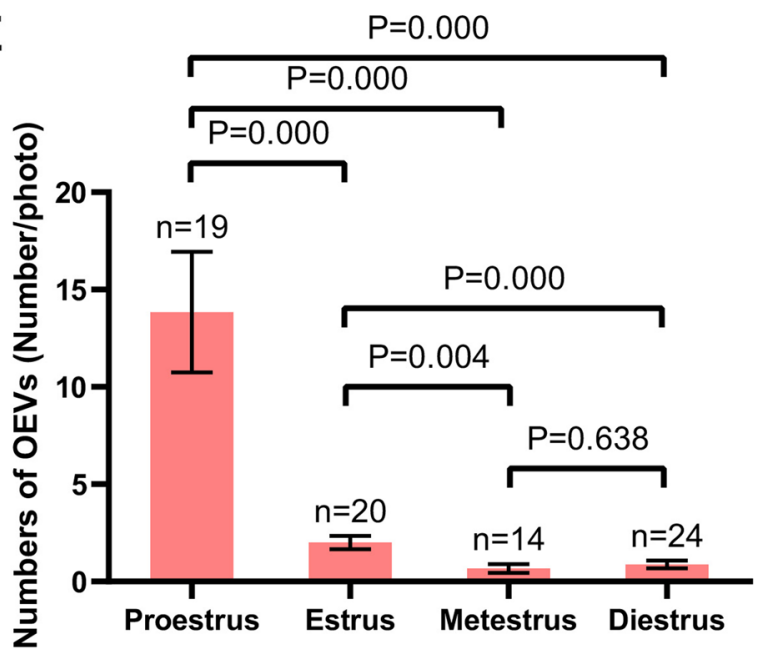

In addition, our results suggest that the natural estrous cycle may be physiologically more beneficial for OEV investigation than the superovulated cycle in the report [5]. On one hand, the natural estrous cycle reflects the physiological status of mice, and the size distribution and the types and numbers of cargoes of the release OEV are not affected by the exogenous hormones, pregnant mare serum gonadotropin (PMSG), and human chorionic gonadotropin (HCG) that 
involved in superovulation. On the other hand, the isolation of OEVs in the natural estrous cycle saves the experimental time and is more convenient than that in the superovulated cycle, because it takes $<1$ day to identify mice using the natural estrous cycle, compared to several days required to achieve superovulation. Admittedly, it is advantageous that the superovulation brings a population of females all into estrus unless special purpose at individual stages in estrus cycle is investigated.

This study clarifies the characteristics and dynamics of OEVs at different stages of the estrous cycle and provides new insights on how to obtain suitable OEVs or extracellular vesicles for potential clinical applications. The clinical application of OEVs may mainly provide the treatments of in vitro fertilization for the higher pregnancy rates in the scenarios of the assistant reproductive technology [11]. The clinical application of OEVs putting into practice still needs to be further developed although OEVs are advantageous to gamete fertilization and embryo development. There are many facing challenges or questions to be addressed: what is the standardization of isolation and purification methods for OEVs; how are the homogenous OEVs with different molecular cargoes obtained; what is the specific molecular cargo in OEVs acting on the recipient cell; what is the target molecule in the recipient cell by acted by OEVs; and what are the signaling networks and mechanisms by which OEVs acts on the recipient cells. Accordingly, this study may offer the clue that the different size distribution of OEVs with different cargoes could be produced and standardized via hormones control.

The limitation of this study has to be noted: the mechanisms by which the size of OEVs differ through the stages of the estrous cycle need to be further revealed in the future, especially which hormone(s) controls the size distribution and cargoes of the released OEVs; only protein cargoes and only three proteins of OEVs through different stages were investigated in the study; and more types and numbers of molecular cargoes of OEVs including proteins, mRNA, siRNA, and lipids need to be further explored in the future.

\section{Conclusions}

In summary, the size distribution characteristics and the ER cargo of OEVs are different and vary across the proestrus, estrus, metestrus, and diestrus stages of the estrous cycle in mice. OEVs are released by the oviduct, which has a dynamic ultrastructure. The results of this study suggest that the size distribution, at least partial protein cargo, and status of released OEVs are influenced by the estrous cycle in mice, which may be associated with sex hormone levels in vivo, although the detailed mechanism requires further study. This study contributes to further understanding the characteristics and dynamics of OEVs at different stages of the estrous cycle; hence, it may be useful for revealing physiological and pathological changes associated with the estrous cycle. The study also provides clues to obtain OEVs or extracellular vesicles with uniform size and stable protein cargoes from other sources, which may have clinical applications.

Acknowledgements The authors wish to thank Mr. Dongwang Zheng for assistance in performing Western blot, animal treatment, and immunoelectron microscopy in this study; Dr. Lu Zhou for assistance with the particle size measurement and TEM; and Ms. Chenyu Yang at Zhejiang University for assistance with the immunoelectron microscopy and TEM of the oviduct.

Funding This study was supported by the Health Sci\&Tech Plan Project of Zhejiang Province (2019KY363), the Sci\&Tech Program Project of Zhejiang Province (2018C37126), Zhejiang Provincial Natural Science Foundation (LY22H040011), the Special Project for the Research Institutions of Zhejiang Province (C11920D-04), and the Zhejiang Provincial Program for the Cultivation of High-level Innovative Health Talents.

Data availability All data generated or analyzed during this study are included in this published article and available from the corresponding author.

Code Availability Not applicable.

\section{Declarations}

Ethics Approval The study protocol was reviewed and approved by the Animal Ethics Committee of Zhejiang Academy of Medical Sciences.

Consent to Participate No patient consent was required for this study.

Consent for Publication The authors and institution agreed with this publication.

Conflict of Interest The authors declare no competing interests.

Open Access This article is licensed under a Creative Commons Attribution 4.0 International License, which permits use, sharing, adaptation, distribution and reproduction in any medium or format, as long as you give appropriate credit to the original author(s) and the source, provide a link to the Creative Commons licence, and indicate if changes were made. The images or other third party material in this article are included in the article's Creative Commons licence, unless indicated otherwise in a credit line to the material. If material is not included in the article's Creative Commons licence and your intended use is not permitted by statutory regulation or exceeds the permitted use, you will need to obtain permission directly from the copyright holder. To view a copy of this licence, visit http://creativecommons.org/licenses/by/4.0/.

\section{References}

1. Simon C, Greening DW, Bolumar D, Balaguer N, Salamonsen LA, Vilella F. Extracellular vesicles in human reproduction in 
health and disease. Endocr Rev. 2018;39:292-332. https://doi.org/ 10.1210/er.2017-00229.

2. Harris EA, Stephens KK, Winuthayanon W. Extracellular vesicles and the oviduct function. Int J Mol Sci. 2020;21. https://doi.org/ 10.3390/ijms21218280.

3. Li K, Ni Y. Progress in the extracellular vesicles regulating maturation and functions of sperm in the reproductive tracts (In Chinese). Chinese Bull Life Sci. 2017;29:562-7. https://doi.org/10. 13376/j.cbls/2017076.

4. de Almeida MMFM, Nagashima JB, Noonan MJ, Crosier AE, Songsasen N. Oviductal extracellular vesicles improve post-thaw sperm function in red wolves and cheetahs. Int J Mol Sci. 2020;21. https://doi.org/10.3390/ijms21103733.

5. Bathala P, Fereshteh Z, Li K, Al-Dossary AA, Galileo DS, Martin-DeLeon PA. Oviductal extracellular vesicles (oviductosomes, OVS) are conserved in humans: murine OVS play a pivotal role in sperm capacitation and fertility. Mol Hum Reprod. 2018;24:14357. https://doi.org/10.1093/molehr/gay003.

6. Asaadi A, Dolatabad NA, Atashi H, Raes A, Van Damme P, Hoelker M, et al. Extracellular vesicles from follicular and ampullary fluid isolated by density gradient ultracentrifugation improve bovine embryo development and quality. Int J Mol Sci. 2021;22. https://doi.org/10.3390/ijms22020578.

7. Banliat C, Le Bourhis D, Bernardi O, Tomas D, Labas V, Salvetti $\mathrm{P}$, et al. Oviduct fluid extracellular vesicles change the phospholipid composition of bovine embryos developed in vitro. Int J Mol Sci. 2020;21. https://doi.org/10.3390/ijms21155326.

8. Bauersachs S, Mermillod P, Alminana C. The oviductal extracellular vesicles' RNA cargo regulates the bovine embryonic transcriptome. Int J Mol Sci. 2020;21. https://doi.org/10.3390/ijms2 1041303.

9. Lopera-Vasquez R, Hamdi M, Fernandez-Fuertes B, Maillo V, Beltran-Brena P, Calle A, et al. Extracellular vesicles from boec in in vitro embryo development and quality. PLoS ONE. 2016;11:e148083. https://doi.org/10.1371/journal.pone.0148083.

10. Fu B, Ma H, Liu D. Extracellular vesicles function as bioactive molecular transmitters in the mammalian oviduct: an inspiration for optimizing in vitro culture systems and improving delivery of exogenous nucleic acids during preimplantation embryonic development. Int J Mol Sci. 2020;21. https://doi.org/10.3390/ ijms21062189.

11. Alminana $\mathrm{C}$, Bauersachs $\mathrm{S}$. Extracellular vesicles in the oviduct: progress, challenges and implications for the reproductive success. Bioengineering (Basel). 2019;6(2). https://doi.org/10.3390/bioen gineering6020032.

12. Caponnetto F, Manini I, Skrap M, Palmai-Pallag T, Di Loreto C, Beltrami AP, et al. Size-dependent cellular uptake of exosomes Nanomedicine-Uk. 2017;13:1011-20. https://doi.org/10.1016/j. nano.2016.12.009.

13. Xu R, Greening DW, Zhu HJ, Takahashi N, Simpson RJ. Extracellular vesicle isolation and characterization: toward clinical application. J Clin Invest. 2016;126:1152-62. https://doi.org/10.1172/ JCI81129.

14. Lee SH, Lira-Albarran S, Saadeldin IM. Comprehensive proteomics analysis of in vitro canine oviductal cell-derived extracellular vesicles. Animals (Basel). 2021;11. https://doi.org/10.3390/ani11 020573.

15. Ferraz M, Carothers A, Dahal R, Noonan MJ, Songsasen N. Oviductal extracellular vesicles interact with the spermatozoon's head and mid-piece and improves its motility and fertilizing ability in the domestic cat. Sci Rep. 2019;9:9484. https://doi.org/10.1038/ s41598-019-45857-x.

16. Al-Dossary AA, Strehler EE, Martin-Deleon PA. Expression and secretion of plasma membrane Ca2+-ATPase 4a (PMCA4a) during murine estrus: association with oviductal exosomes and uptake in sperm. PLoS ONE. 2013;8:e80181. https://doi.org/10. 1371/journal.pone.0080181.

17. Byers SL, Wiles MV, Dunn SL, Taft RA. Mouse estrous cycle identification tool and images. PLoS ONE. 2012;7:e35538. https:// doi.org/10.1371/journal.pone.0035538.

18. McLean AC, Valenzuela N, Fai S, Bennett SA. Performing vaginal lavage, crystal violet staining, and vaginal cytological evaluation for mouse estrous cycle staging identification. J Vis Exp. 2012:e4389. https://doi.org/10.3791/4389.

19. Murray MK, DeSouza MM, Messinger SM. Oviduct during early pregnancy: hormonal regulation and interactions with the fertilized ovum. Microsc Res Tech. 1995;31:497-506. https://doi.org/ 10.1002/jemt.1070310606.

20. Barton BE, Herrera GG, Anamthathmakula P, Rock JK, Willie A, Harris EA, et al. Roles of steroid hormones in oviductal function. Reproduction. 2020;159:R125-37. https://doi.org/10.1530/ REP-19-0189.

21. Wang H, Eriksson H, Sahlin L. Estrogen receptors alpha and beta in the female reproductive tract of the rat during the estrous cycle. Biol Reprod. 2000;63:1331-40. https://doi.org/10.1095/biolreprod 63.5.1331.

22. Caballero JN, Frenette G, Belleannee C, Sullivan R. CD9-positive microvesicles mediate the transfer of molecules to bovine spermatozoa during epididymal maturation. PLoS ONE. 2013;8:e65364. https://doi.org/10.1371/journal.pone.0065364.

23. Théry C, Zitvogel L, Amigorena S. Exosomes: composition, biogenesis and function. Nat Rev Immunol. 2002;2(8):569-79. https://doi.org/10.1038/nri855.

24. Alminana C, Tsikis G, Labas V, Uzbekov R, Da Silveira JC, Bauersachs S, et al. Deciphering the oviductal extracellular vesicles content across the estrous cycle: implications for the gametes-oviduct interactions and the environment of the potential embryo. BMC Genomics. 2018;19:622. https://doi.org/10.1186/ s12864-018-4982-5.

25. Klohonatz KM, Cameron AD, Hergenreder JR, Da Silveira JC, Belk AD, Veeramachaneni DN, et al. Circulating miRNAs as potential alternative cell signaling associated with maternal recognition of pregnancy in the mare. Biol Reprod. 2016;95:124. https://doi.org/10.1095/biolreprod.116.142935.

26. Serrano-Pertierra E, Oliveira-Rodríguez M, Rivas M, Oliva P, Villafani J, Navarro A, et al. Characterization of plasma-derived extracellular vesicles isolated by different methods: a comparison study. Bioengineering. 2019;6(1):8. https://doi.org/10.3390/bioen gineering6010008.

27. Li K, Xue Y, Chen A, Jiang Y, Xie H, Shi Q, et al. Heat shock protein 90 has roles in intracellular calcium homeostasis, protein tyrosine phosphorylation regulation, and progesterone-responsive sperm function in human sperm. PLoS ONE. 2014;9:e115841. https://doi.org/10.1371/journal.pone.0115841.

28. Hamilton KJ, Arao Y, Korach KS. Estrogen hormone physiology: reproductive findings from estrogen receptor mutant mice. Reprod Biol. 2014;14:3-8. https://doi.org/10.1016/j.repbio.2013.12.002.

29. Levin ER. Plasma membrane estrogen receptors. Trends Endocrinol Metab. 2009;20:477-82. https://doi.org/10.1016/j.tem. 2009.06.009.

30. Rappa G, Santos MF, Green TM, Karbanova J, Hassler J, Bai Y, et al. Nuclear transport of cancer extracellular vesicle-derived biomaterials through nuclear envelope invagination-associated late endosomes. Oncotarget. 2017;8:14443-61. https://doi.org/ 10.18632/oncotarget.14804.

31. Mathivanan S, Ji H, Simpson RJ. Exosomes: extracellular organelles important in intercellular communication. J Proteomics. 2010;73:1907-20. https://doi.org/10.1016/j.jprot.2010.06.006.

32. Gatien J, Mermillod P, Tsikis G, Bernardi O, Janati Idrissi S, Uzbekov R, et al. Metabolomic profile of oviductal extracellular 
vesicles across the estrous cycle in cattle. Int J Mol Sci. 2019;20. https://doi.org/10.3390/ijms20246339.

33. Gill S, Catchpole R, Forterre P. Extracellular membrane vesicles in the three domains of life and beyond. FEMS Microbiol Rev. 2019;43:273-303. https://doi.org/10.1093/femsre/fuy042.

34. Margolis L, Sadovsky Y. The biology of extracellular vesicles: The known unknowns. Plos Biol. 2019;17:e3000363. https://doi. org/10.1371/journal.pbio.3000363.

35. Gonzalez E, Piva M, Rodriguez-Suarez E, Gil D, Royo F, Elortza $F$, et al. Human mammospheres secrete hormone-regulated active extracellular vesicles. PLoS ONE. 2014;9:e83955. https://doi.org/ 10.1371/journal.pone.0083955.

36. Walmer DK, Wrona MA, Hughes CL, Nelson KG. Lactoferrin expression in the mouse reproductive tract during the natural estrous cycle: correlation with circulating estradiol and progesterone. Endocrinology. 1992;131:1458-66. https://doi.org/10.1210/ endo.131.3.1505477.

37. Parkening TA, Collins TJ, Smith ER. Plasma and pituitary concentrations of $\mathrm{LH}, \mathrm{FSH}$, and prolactin in aging C57BL/6 mice at various times of the estrous cycle. Neurobiol Aging. 1982;3:31-5. https://doi.org/10.1016/0197-4580(82)90058-6.

38. Sarkar DK, Chiappa SA, Fink G, Sherwood NM. Gonadotropin-releasing hormone surge in pro-oestrous rats. Nature. 1976;264:461-3. https://doi.org/10.1038/264461a0.

39. Rajendren G, Gibson MJ. A confocal microscopic study of synaptic inputs to gonadotropin-releasing hormone cells in mouse brain: regional differences and enhancement by estrogen. Neuroendocrinology. 2001;73:84-90. https://doi.org/10.1159/000054624.

40. Hawkins SM, Matzuk MM. The menstrual cycle: basic biology. Ann N Y Acad Sci. 2008;1135:10-8. https://doi.org/10.1196/ annals.1429.018.
41. Montgomery V, Loutradis D, Tulchinsky D, Kiessling A. FSHinduced ovulation in intact and hypophysectomized mice. J Reprod Fertil. 1988;84:1-6. https://doi.org/10.1530/jrf.0.08400 01.

42. Kumar TR, Wang Y, Lu N, Matzuk MM. Follicle stimulating hormone is required for ovarian follicle maturation but not male fertility. Nat Genet. 1997;15:201-4. https://doi.org/10.1038/ ng0297-201.

43. Appelgren LE. Histochemical demonstration of drug interference with progesterone synthesis. J Reprod Fertil. 1969;19:185-6. https://doi.org/10.1530/jrf.0.0190185.

44. Sander VA, Facorro GB, Piehl L, Rubin De Celis E, Motta AB. Effect of DHEA and metformin on corpus luteum in mice. Reproduction. 2009;138:571-9. https://doi.org/10.1530/REP-08-0325.

45. Rudolph M, Docke WD, Muller A, Menning A, Rose L, Zollner $\mathrm{TM}$, et al. Induction of overt menstruation in intact mice. PLoS ONE. 2012;7:e32922. https://doi.org/10.1371/journal.pone.00329 22.

46. Stocco C, Telleria C, Gibori G. The molecular control of corpus luteum formation, function, and regression. Endocr Rev. 2007;28:117-49. https://doi.org/10.1210/er.2006-0022.

47. Morita M, Sugimoto M, Miyamoto H, Manabe N. Ultrastructural features of secretion by murine oviductal epithelium. J Mamm Ova Res. 1997;14:72-8. https://doi.org/10.1274/jmor.14.72.

48. Noreikat K, Wolff M, Kummer W, Kolle S. Ciliary activity in the oviduct of cycling, pregnant, and muscarinic receptor knockout mice. Biol Reprod. 2012;86:120. https://doi.org/10.1095/biolr eprod.111.096339. 